\section{Judgment of short time intervals while performing mathematical tasks*}

\author{
WILLIAM BURNSIDE \\ Indiana University, Bloomington, Indiana 47401
}

Ornstein (1969) suggested that the apparent duration of a time interval depended upon the memory storage size. This experiment tests this theory by studying duration estimates made while performing different types of information processing. Twenty Ss, introductory psychology students, served in each of the 30 conditions of the experiment. The six time intervals estimated by the method of reproduction were of lengths of 10,12 , $14,16,18$, and $20 \mathrm{sec}$, and the five intervening tasks were doing nothing, reading numbers, adding, multiplying, and adding and multiplying in a random order. The results show that fewer items were output in the three arithmetic conditions, as compared with the reading condition, and also show that the duration estimates were shorter for these three conditions. Estimates of duration are related to amount of output, and also appear to be related to the type of processing done to produce this output.

Many philosophers, including Kant, have agreed that the origin of our idea of time is change, and that time is an activity of the mind resulting from observation of successive changes. In his review of psychological studies of the experience of time, Fraisse (1963) agrees with this viewpoint by stating: "The birth of the notion of time is no doubt the result of the experience of successions, of which some are periodic and others not, of continuous and discontinuous changes, of interwoven renewals and relatively permanent states." Guyau (1890) was the first to relate this viewpoint of time experience to human memory processes by stating that changes are registered as a series of images in the brain, in which more distant images are obliterated by closer more distinct ones. Recent work on human memory processes provides an improved foundation for the study of how temporal judgments may depend upon memory processes. Examples of this work are Miller's (1956) concept of "chunking" or coding of items in memory, Peterson and Peterson's (1959) study of short-term memory, and Atkinson and Shiffrin's (1968) distinction between shortand long-term stores.

Ornstein (1969), in his storage-size theory, argues that the experience of duration of an interval is a construction formed from the size of storage occupied by memories of that interval, not all memories put into store during the interval, but only those retrievable from store at the time the duration estimate is made. Increasing or decreasing the input, altering the coding or "chunking" of the input, forgetting, or anything else affecting the memory of an interval after it is over, will also affect the experience of duration

*The assistance of Drs. Frank Restle and Richard Shiffrin is gratefully acknowledged. of that interval. Ornstein has reported experiments which lend support to his theory, showing that Ss' experience of the duration of an interval increases as the stimuli presented to him during that interval become more complex, and that coding of the occurrences during an interval will decrease the experience of the duration of that interval. The storage size of an interval can be reduced by means of different levels of coding or forgetting.

Ornstein has presented evidence of a relationship between time experience and size of memory store by considering the number of items input and their complexity, and possible coding of these items while in store. But it is difficult to determine the size of memory store for a given interval. Storage size could be related to the number of items input during the interval, the number of items output on a memory test after the interval has passed, or any processing done to transform the input items into different storage items. Storage size would seem to depend upon whether the substeps in any required store, or whether only the answers reached by the processing are retained. In Ornstein's experiments, Ss passively received stimuli either visually or auditorially, or performed simple tasks such as pursuit rotor training or verbal learning. It is important to extend Ornstein's research and consider tasks that require higher levels of information processing. Consideration of information processing may lead to clarification of a problem discussed by Gilliland, Hofeld, and Eckstrand (1946). Periods of time in which we experience little or no incoming stimulation often seem unbearably long and are labeled as boring, while time often seems to go by very rapidly when one is deeply involved in thought about a information processing are retained in problem in which he is interested. Neither of these experiences seems to fit with a theory considering only the number of items input or output as comprising memory store.

Ornstein related the temporal judgment of the length of an interval to the size of memory store occupied by memories of that interval, but he does not consider the effects of different levels of information processing upon this storage size. In a task such as arithmetic, increased processing per problem leads to fewer solutions in a given time interval. Multiplication problems require more processing for solution than do addition problems, and thus fewer solutions are reached in a given time interval for the multiplication problems. If the processing substeps are discarded from memory store and only the solutions are retained, intervals requiring a lot of processing for each solution would be judged as shorter than intervals in which little processing is done. This is reasonable because more items could be placed in store during the latter intervals. If the processing substeps are retained in store, intervals in which Ss are working as rapidly as possible should be judged as being the same length, regardless of the level of information processing done during the interval. The present experiment employs different types of arithmetic problems to study the effects of different levels of information processing upon duration judgments, and to relate these effects to size oí memory store.

\section{METHODS}

\section{Subjects}

The Ss were 20 Indiana University introductory psychology students, 12 female and 8 male, who participated in the experiment as a part of their course requirements, and who had no knowledge of the experiment before they participated.

\section{Apparatus}

The experiment was conducted in a small room in which there were no clocks and no rhythmic sounds that might have served the Ss as an aid in timing. Six intervals were used. These intervals, 10,12 , $14,16,18$, and $20 \mathrm{sec}$, were timed by means of a Hunter timer, and a buzzer was wired to this timer so that it sounded briefly whenever the required interval timed out. A clock, to which a toggle switch was attached by a long cord, was placed on the table so that its face could be seen by E, but not by the Ss.

A deck of $305 \times 7$ in. cards was used for displaying mathematical problems, one card for each condition. The Ss solved these without the aid of a pencil and paper. Six of these cards were blank. Six cards 


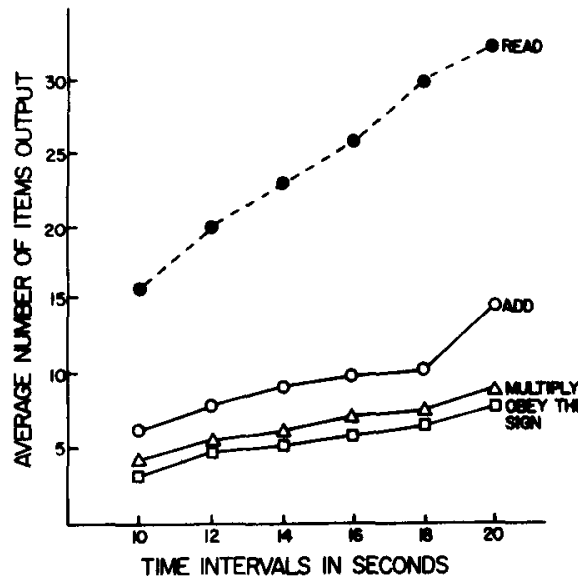

Fig. 1. Number of numeric items output during six different time intervals.

had the word "Read" printed at the top and below this were 30 two-digit numbers spaced in five uneven rows. The remaining cards each displayed 30 one-digit by two-digit mathematical problems, with six cards having the word "Add" printed at the top, six having the word "Multiply," and six having the words "Obey the Sign." The latter six cards required addition and multiplication in a random order, the multiplication problems being indicated with an " $x$ " beside the problem. All the numbers were chosen from a random-numbers table. with the restrictions that the one-digit numbers in the addition problems could not be zero, the one-digit numbers in the multiplication problems could not be zero or one, and the answers to all problems be two-digit numbers. The Ss' time estimates and problem solutions were recorded manually by E.

\section{Procedure}

Each $S$ sat at a table opposite $E$ and removed his watch prior to the experiment. After being shown an example of each of the five types of cards to be used, each $S$ immediately began performing the task indicated at the top of a card when $\mathrm{E}$ turned the card over (do nothing, read, add, multiply, and add and multiply), and continued performing the task until he heard the buzzer sound. He then flipped the clock switch on and did nothing for a period he felt to be equivalent to the time he had just spent responding. He then flipped the switch off. Thus, the estimate was made by the method of reproduction. This procedure was followed for 30 trials for each $S$, with the $S$ s going through the numbers or problems on the cards as rapidly as possible, while still saying the numbers or answers clearly. The Ss looked at the blank card and said nothing in the blank condition.

Each $\mathrm{S}$ was presented a total of 30 cards,
6 for each of the conditions (blank, read, add, multiply, and obey the sign). All 30 cards were shuffled for a given group of four Ss, so order of presentation was completely random in each session. Each interval was used exactly once in each condition, the order of interval lengths being randomized within a condition. A new randomization was used for each group of four Ss. There was a short delay of 3 or $4 \mathrm{sec}$ after each reproduction estimate, while $E$ recorded the estimate and set the timer dials for the next interval to be judged. As accurately as possible, $\mathrm{E}$ turned the next card face up on each trial at the same instant that he switched the timer on. When the buzzer sounded, E quickly removed the card and $S$ immediately began his reproduction interval. Estimates were recorded to the nearest 10 th of a second, and, in the read condition, the number of numbers read on each trial was recorded, while in the arithmetic conditions all of each $S$ 'S answers were recorded.

\section{RESULTS}

During the intervals presented, the add answers were $97 \%$ correct, the multiply answers were $91 \%$ correct, and the obey answers were $92 \%$ correct. No such data were collected for the read condition, but it was observed that the Ss were highly accurate in reading numbers at high speeds. Thus, it is safe to assume that Ss did perform the assigned tasks during the presentation intervals.

The average numbers of overt responses output are shown in Fig. 1, which shows that there was much more output in the read condition than in the multiply and obey conditions. The average reproduction intervals produced by $20 \mathrm{Ss}$ for each of the 30 conditions in the experiment are shown in Fig. 2; reproduction intervals were slightly longer than presentations in the blank and read conditions, but were shorter in the other three conditions. An analysis of variance indicated that types of intervening tasks had a significant effect $(\mathrm{F}=21.823, \mathrm{df}=4 / 570, \quad \mathrm{p}<.01), \quad$ and Fig. 2 indicates that most of this difference is between the blank and read conditions and the other three conditions.

\section{DISCUSSION}

Ornstein (1969), in his experiments, went to great lengths to conceal from his Ss the fact that they were participating in a time-estimation experiment, for he felt that otherwise the $\mathrm{Ss}$ would count to improve their timing. Such concealment was unnecessary in the present study since the $\mathrm{Ss}$ were responding with numbers during the presentation intervals. The Ss may have counted to themselves in the

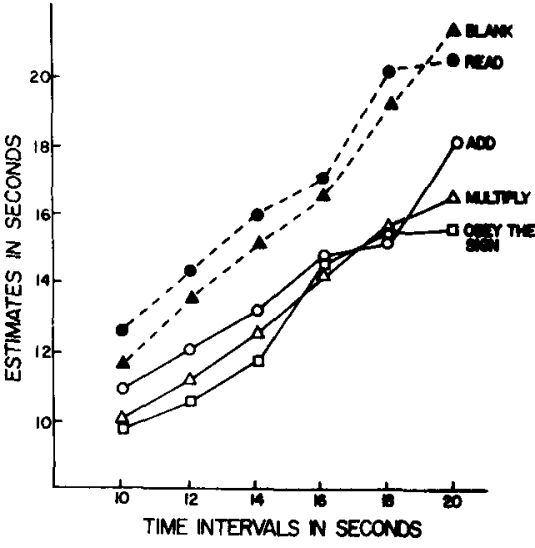

Fig. 2. Duration estimates made during five different intervening tasks.

blank conditon, but this condition was included in order to find how accurate $S$ s are when using any personal means they choose for timing.

If one assumes that a small amount of output represents a small amount of storage (this could be tested with a memory test in a future experiment), then Figs. 1 and 2 offer support for Ornstein's storage-size theory. Duration estimates decreased under those conditions in which fewer responses were made, with the biggest gap in both graphs being between the read condition and the three arithmetic conditions. Only small differences were found between the three arithmetic conditions, since the multiplication problems were very simple. That is, the answers to these problems were two-digit numbers. The only data that seem at first glance troublesome for a storage-size theory are the estimates under the blank condition, which are slight overestimates. But these data can be accounted for if one considers the $S s$ as providing self-stimulation in the blank condition, by counting or some other means.

Suppose that the read condition requires more information processing than the blank condition, add more processing than read, multiply more than add, and obey most of all, since the Ss could not develop a set for one type of operations in this condition. Then estimates of duration in this experiment were found to decrease as amount of necessary information processing increased. We do not know that it requires more processing to multiply 5 numbers than to read 15 numbers, but data such as those presented by Peterson (1969) indicate that Ss can perform a concurrent task while reading numbers, but not while problem solving. This supports an argument for different levels of information processing occurring in this experiment. However, since the Ss were working as quickly as possible in each 
condition, the only valid argument that can be made is that Ss were processing at full capacity in each condition, but were performing different types of processing. In terms of a storage-size theory, the data indicate that duration estimates decreased as storage size decreased, with items output making up the storage and substeps in the information processing being discarded.

A theory considering only items output and not information processing as determining storage size would predict that a duration estimate would depend upon the number of numbers in memory store, regardless of what mathematical process was used to generate those numbers. It can be seen from Fig. 1 that adding for $10 \mathrm{sec}$ and multiplying for $18 \mathrm{sec}$ both resulted, on the average, in about six answers being generated. Considering items output as a good measure of size of memory store, a storage-size theory would predict that the estimates of these intervals would be approximately the same. Fig. 2 shows that the average add-10 estimate was about $11 \mathrm{sec}$ and the average multiply-18 estimate was about $15.75 \mathrm{sec}$. Evidently, after performing a task, Ss have in store not only the number of items completed, but also some memory of the type of task done to get those items. Thus, it seems that even though processing substeps are apparently discarded from memory store, the type of information processing done has an effect upon duration judgments.
Time estimations may be made by considering the number of individual tasks performed during the interval estimated, and then transforming this number to a temporal estimate by considering the type of processing done for each task. Durations filled with mathematical problems appear shorter than intervals filled with less demanding activity, however, so the corrections for duration of the individual tasks are imperfect. This may be because the substeps in performing arithmetic are stored in short-term memory and may be lost before the result is stored in more stable form, to be remembered at the time of interval reproduction. The possibility that different memory demands result in different short-term forgetting curves has recently been demonstrated in a different context (Restle, 1970).

This experiment indicates that duration judgments depend upon the number of items in memory store, as measured by number of items output, and that information-processing substeps are not retained in store. However, it also indicates that a memory of the type of processing done is retained in store, and may affect temporal judgments, since intervals in which a lot of processing was done seemed shorter. The distinction between amount remembered about an interval (which leads to long apparent duration) and amount of information processed during an interval, helps to clarify the familiar paradox that time seems to pass either very quickly or very slowly when one is exceptionally busy. Time may seem to pass quickly when a lot of processing is done to obtain a few solutions, but it may seem to pass slowly while one is performing a tedious task in which all the substeps are remembered.

\section{REFERENCES}

ATKINSON, R. C., \& SHIFFRIN, R. M. Human memory: A proposed system and its control processes. In K. W. Spence and J. T. Spence (Eds.), The psychology of learning and motivation: Advances in research and theory. Vol. 2. New York: Academic Press, 1968.

FRAISSE, P. The psychology of time. New York: Harper \& Row, 1963.

GILLILAND, A. R., HOFELD, J., \& ECKSTRAND, G. Studies in time perception. Psychological Bulletin, 1946, 43, 162-176.

GUYAU, M. La Genese de l'idee de temps, Alcan, 1890. Cited in R. E. Ornstein, On the experience of time. Middlesex, England: Penguin Books, 1969.

MILLER, G. A. Magical number 7, plus or minus two. Psychological Review, 1956, 63, 81-97. ORNSTEIN, R. E. On the experience of time. Middlesex, England: Penguin Books, 1969.

PETERSON, L. R. Concurrent verbal activity. Psychological Review, 1969, 76, 376-386.

PETERSON, L. R., \& PETERSON, M. J. Short-term retention of individual verbal items. Journal of Experimental Psychology, 1959, 58, 193-198.

RESTLE, F. Training of short-term memory. Journal of Experimental Psychology, 1970, 83, 224-226.

(Accepted for publication July 6, 1970.) 